\title{
Polymer multilayer film formation studied by in situ ellipsometry and electrochemistry ${ }^{\text {is }}$
}

\author{
Karolina Haberska a,b,*, Tautgirdas Ruzgas ${ }^{\text {a }}$ \\ a Biomedical Laboratory Science and Technology, Faculty of Health and Society, Malmö University, SE-205 06 Malmö, Sweden \\ b Department of Analytical Chemistry, Lund University, P.O. Box 124, SE-221 00 Lund, Sweden
}

\section{A R T I C L E I N F O}

\section{Article history:}

Received 22 January 2009

Received in revised form 10 May 2009

Accepted 20 May 2009

Available online 27 May 2009

\section{Keywords:}

Polyelectrolyte multilayers

Layer-by-Layer

Self assembly

Pinhole model

Membrane model

\begin{abstract}
A B S T R A C T
Polyelectrolyte multilayer films adsorbed on gold surfaces were studied by combined ellipsometric and electrochemical methods. Multilayers were composed of "synthetic" (poly(4-styrenesulfonic acid) ammonium salt (PSS) and poly(allylamine hydrochloride) (PAH) (PSS/PAH)) and "semi-natural" (carboxymethyl cellulose $(\mathrm{CMC})$ and chitosan $(\mathrm{CHI})(\mathrm{CMC} / \mathrm{CHI})$ ) polyelectrolytes. It was found that only PSS/PAH Layer-by-Layer (LbL) assembled structures result in dense surface confined films that limit permeability of small molecules, such as ferri-/ferrocyanide. The PSS/PAH assemblies can be envisaged as films with pinholes, through which small molecules diffuse. During the LbL deposition process of these films a number of pinholes quickly decay. A representative pinhole diameter was found to be approximately $20 \mu \mathrm{m}$, which determines the diffusion of small molecules through LbL films, and yet remains constant when the film consists of a few LbL assembled polyelectrolyte bilayers. $\mathrm{CMC} / \mathrm{CHI} \mathrm{LbL}$ assemblies at gold electrode surfaces give very low density films, which do not limit the diffusion of ferri-/ferrocyanide between the surface of the electrode and the solution.
\end{abstract}

(c) 2009 Elsevier B.V. All rights reserved.

\section{Introduction}

Polyelectrolyte multilayers assembled from oppositely charged polymers have gained a considerable scientific interest inspired by possible application in the fields of, e.g., biosensors [1], electronic devices [2], drug delivery carriers [3], micropatterning [4], membranes [5], and microcontainers for molecules encapsulation [6]. The multilayer construction technique using Layer-by-Layer (LbL) deposition procedure was first introduced by Decher [7] in the beginning of 1990s and initially realised on solid surfaces. Lately, the thin films were applied on almost any type of charged substrates regardless its shape and origin including even enzymes [8], proteins [9], colloidal particles [10], and nanoparticles [11]. Furthermore, incorporation of the materials with functionalities, such as biomolecules, cells [12], dendrimers [13], and inorganic nanoparticles [14] into polyelectrolyte

Abbreviations: $\mathrm{CHI}$, chitosan; $\mathrm{CMC}$, sodium carboxymethyl cellulose; $\mathrm{CV}$, cyclic voltammogram; HA, hyaluronic acid; LbL, Layer-by-Layer; PAA, poly(acrylic acid); $\mathrm{PAH}$, poly(allylamine hydrochloride); PDADMAC, poly(diallyldimethylammonium); PEI, poly(ethyleneimine); PGA, poly(l-glutamic acid); PLL, poly(l-lysine); PSS, poly (4-styrenesulfonic acid) ammonium salt; Tris, tris(hydroxymethyl)aminomethane.

is Dedicated to Professor Lo Gorton on the occasion of his 60th birthday.

* Corresponding author. Department of Analytical Chemistry, Lund University, P.O. Box 124, SE-221 00 Lund, Sweden. Tel.: +46 76828 3664; fax: +46 406658100.

E-mail address: karolina.haberska@mah.se (K. Haberska). multilayers significantly broadened properties and potential applications of the polymer films.

The main driving force of multilayer self assembly is electrostatic attraction between oppositely charged polymers [15] carrying a minimum charge density depending on the polymer system [16], i.e., $20 \%$ of charged blocks per chain could be sufficient for multilayer formation [17]. In addition, short-range secondary interactions (hydrophobic, hydrogen-bonding) and steric repulsions determine the multilayer structure. Long- and short-range interactions usually lead to charge reversal of the multilayer after each deposition step [18].

Initially, the architecture of the polyelectrolyte films has been thought as a lamella structure, consisting of individual molecular layers, where polyanions and polycations intimately interdigitate one another [19]. However, later it was shown that LbL assemblies are dynamic and not perfectly well defined multi-composite systems, with a diffuse change between interpenetrating layers [20]. In case of adjacent, flexible layers of poly(styrene sulfonate) (PSS) and poly (allylamine hydrochloride) (PAH) internal interface roughness measured by neutron reflectometry [21] and X-ray reflectivity [22] were found to be in the order of $2 \mathrm{~nm}$. Amorphous nature of the polymer multilayers has been extensively studied also by scanning electron and atomic force microscopy [23], confocal spectroscopy [24], focused ion bean [25], surface plasma resonance [26], quartz crystal microbalance [27], ellipsometry [28], and electrochemistry [29]. 
Self assembly process provides a possibility to adjust and control thickness of the film with nanometre precision, inter alia, by adding salt to the polyion solutions [30]. The layer thickness increases with the ionic strength of the dipping solution [31], and within the concentration range 0.5 to $3 \mathrm{M}$ grows linearly in case of PSS/PAH system [21]. Polymer film development, in terms of mass and thickness with the number of deposition steps, proceeds either in linear or exponential manner depending on ionic strength of the polyelectrolyte solution, charge density, and $\mathrm{pH}$ (in case of weak polyacids or polybases) [32,33]. The best known linearly growing systems with 1 to $10 \mathrm{~nm}$ thickness per layer are composed of PSS/PAH [34], poly (diallyldimethylammonium) (PDADMAC)/PSS [35] or poly (acrylic acid) (PAA)/PAH [32] polymers. Exponentially increasing films consist of natural polyelectrolytes and poly(amino acid)s, i.e., poly(L-lysine) (PLL)/hyaluronic acid [36], poly(L-glutamic acid) (PGA)/PLL [37], and PGA/PAH [27]. They proved to be much less structured and highly hydrated films, behaving as a polycation/ polyanions gels, and partly due to their bio-mimicking properties suitable for biomaterial coatings [38]. The exponential growth of multilayers is highly linked to vertical (normal to the surface) mobility of polyelectrolytes within layers, e.g. PGA/PLL assemblies, and diffusion "in" and "out" through the whole structure during each double layer deposition step [39]. Moreover, lateral mobility of polymers chains in multilayer matrix was also observed and controlled by the interaction forces between neighbouring polyelectrolyte stratum, and by swelling features of the polymer complexes [40]. The structure of the multilayers is ruled by the primary adsorption conditions with variation between the newly adsorbed part and re-swollen precursor part of the films. The water content of the already established layers is about $10 \%$ lower than in the freshly adsorbed layers, where the water volume fraction is close to $56 \%$, which causes a gradient in density across the multilayers system [31]. The mass density of multilayers depends on a complex relationship between the charge density, intrinsic chain rigidity, and hydrophobic/hydrophilic balance [41]. The above mentioned parameters are particular for the chemical structure of polyelectrolytes, though capable of being regulated by $\mathrm{pH}$ for weak polyacids or polybases. Besides the density impact, the permeability of polymer films can be adjusted with the number of assembled polyelectrolyte layers [42] and with the solution concentration of salts [43]. The understanding and control of multilayer porosity provide appealing ways of controlled release of encapsulated compound, and open up new applications of polyelectrolyte films.

Our work focuses on the investigation the diffusion properties and permeability of polymer multilayers, and evaluation of the theoretical models describing mass transport of solution species through the film. The "pinhole model" describes polymer as an impenetrable layer comprising of many small pinholes serving as a channels for the penetrating molecules. The multilayer can also be considered as a homogeneous phase ("membrane model"), through which a homogeneous diffusion of different species occurs [44]. When the number of layers increases simultaneously with the thickness of the film the diffusion process transforms from linear to radial and the multilayers could be envisage as an array of microelectrodes. To distinct between these two mentioned models electrochemical techniques, specifically, chronoamperometry and cyclic voltammetry can be used $[45,46]$. Another frequently used method to study multilayers development on solid surfaces is ellipsometry. We have combined electrochemical and ellipsometric methods to study the structure of polymer multilayers, as application of these joined methods have only been subject to limited research $[47,48]$.

The present paper concentrates on two polyelectrolyte systems chosen to acquire properties of multilayer films based on "totally artificial" and "semi-natural" polymers. Well described in the literature multilayer model system based on synthetic polymers: PSS/PAH has been compared to a polysaccharide system: carbox- ymethyl cellulose (CMC) and chitosan ( $\mathrm{CHI})(\mathrm{CMC} / \mathrm{CHI})$. The polymer multilayers have been prepared by the LbL technique on gold surfaces modified with poly(ethylene imine) (PEI). One of the multilayer formation parameters, i.e., the adsorption and rinsing medium, has been varied in order to understand the effect on the multilayer thickness, the adsorbed amount, and the structure in respect to diffusion properties of small charged redox molecule, i.e. the ferri-/ ferricyanide ion.

\section{Experimental}

\subsection{Reagents and solutions}

Poly(ethylene imine) (PEI) (50\% w/v in water; $\left.M_{\mathrm{w}} \approx 750000\right)$, poly(4-styrenesulfonic acid) ammonium salt (PSS) (30\% wt. in water; $\left.M_{\mathrm{w}} \approx 200000\right)$, poly(allylamine hydrochloride) $(\mathrm{PAH})\left(M_{\mathrm{w}} \approx 70000\right)$, sodium carboxymethyl cellulose $(\mathrm{CMC})\left(M_{\mathrm{w}} \approx 90000\right)$, chitosan (CHI) from crab shells, (low-viscosity; $M_{\mathrm{w}} \approx 750000$ ), $\mathrm{NaH}_{2} \mathrm{PO}_{4}$, $\mathrm{Na}_{2} \mathrm{HPO}_{4}, \mathrm{NaCl}, \mathrm{KCl}, \mathrm{H}_{2} \mathrm{SO}_{4}, \mathrm{~K}_{4}\left[\mathrm{Fe}(\mathrm{CN})_{6}\right]$, citric acid, and buffer salt tris(hydroxymethyl)aminomethane (Tris) were used as received from Sigma-Aldrich Sweden AB (Stockholm, Sweden). Deionised water (18 MW, PURELAB ${ }^{\mathrm{TM}}$ ELGA LabWater, UK) was used for preparation of all solutions and for cleaning procedures. Polyelectrolytes used for preparing PSS/PAH films were dissolved in $20 \mathrm{mM}$ Tris buffer with $100 \mathrm{mM} \mathrm{KCl}, \mathrm{pH}$ 7.8, whereas polysaccharides, CMC/CHI, in $50 \mathrm{mM}$ citrate-phosphate buffer with $50 \mathrm{mM} \mathrm{NaCl}$, pH 5.5. In case of chitosan, all adsorption solution contained additionally 1 vol.\% of acetic acid. Furthermore, solutions for electrochemical studies contained $4 \mathrm{mM} \mathrm{K}_{4}$ $\left[\mathrm{Fe}(\mathrm{CN})_{6}\right]$ in the above noted buffers.

Due to the different buffers used the influence of the type of salts on the properties of the adsorbed films both during the process and on established layers in terms of permeability to the redox probe was also investigated. No significant difference was found between the films made in the presents of either $\mathrm{Na}^{+}$or $\mathrm{K}^{+}$ions. This is in agreement with the previous report stating that the thickness of the PAH/PSS layers is independent on the kind of salt $(\mathrm{NaCl}$ or $\mathrm{KCl})$. However the polyelectrolyte multilayers adsorbed from $\mathrm{NaCl}$ solutions might have more bound water [49].

\subsection{Ellipsometry}

The multilayer film formation was monitored using in situ ellipsometry, an optical method based on measuring changes in the polarization of the light reflected at a planar surface. An automated ellipsometer (type 43603-200E, Rudolph Research, Fairfield, NJ, USA) was equipped with a xenon lamp of a fixed angle of incidence $\left(67.83^{\circ}\right)$ and the light detector (442.90 nm) with an interference filter (ultraviolet and infrared blocking, Melles Griot, Netherlands). The substrates for ellipsometric studies were manufactured in a Balzers UMS 500 P system by electron-beam deposition of $200 \mathrm{~nm}$ of gold onto silicon (100) wafers, precoated with a 2.50-nm-thick titanium adhesion layer (Laboratory of Applied Physics, Linköping University, Sweden). Prior to each experiment the gold surface was cleaned electrochemically for $30 \mathrm{~min}$ in $500 \mathrm{mM} \mathrm{H}_{2} \mathrm{SO}_{4}$ by means of cyclic voltammetry, i.e., by sweeping the applied potential between 0 and 1.9 vs. NHE with scan rate of $100 \mathrm{mV} \mathrm{s}^{-1}$ for $30 \mathrm{~min}$. Next, the surface was vertically mounted into a glass trapezoid cuvette (Hellma, Germany) containing $5 \mathrm{ml}$ of solution thermostated at $25{ }^{\circ} \mathrm{C}$ and equipped with magnetic stirrer. Additional specification of the ellipsometer setup is described by Landgren and Jönsson [50]. In order to determine the refractive index of the gold surface, prior to each measurement, a twozone surface characterization in solution was carried out. After a stable baseline was obtained, polymers from stock solutions were added to the cuvette and the change in ellipsometric angels were recorded in situ every 15 th second. The adsorbed amount $(\Gamma)$ on the surface was calculated from the time-determined values of the mean optical 
thickness $\left(d_{\mathrm{f}}\right)$ and the refractive index $\left(n_{\mathrm{f}}\right)$ of the layers according to Feijter formula (Eq. (1)), where $n_{\text {medium }}$ is the refractive index of the adsorption solution [51]:

$\Gamma=d_{\mathrm{f}} \frac{n_{\mathrm{f}}-n_{\text {medium }}}{d n / d c}$

The refractive index increment value $(\mathrm{d} n / \mathrm{d} c)$ of $0.196 \mathrm{ml} \mathrm{g}^{-1}$ was used, as reported by Caruso et al. [52], for PSS/PAH multilayers, whereas $0.17 \mathrm{ml} \mathrm{g}^{-1}$ for $\mathrm{CMC} / \mathrm{CHI}$ film averaged from values determined for CMC $0.15 \mathrm{ml} \mathrm{g}^{-1}$ [53] and $0.19 \mathrm{ml} \mathrm{g}^{-1}$ for chitosan [54]. Precursor layer of polyethyleneimine ( $1 \mathrm{mg} \mathrm{ml}^{-1}$ ) for both studied systems was adsorbed for $5 \mathrm{~min}$ on the bare gold surface immersed in buffer or water. After that the cuvette was rinsed for $5 \mathrm{~min}$ with relevant solution at a continuous flow of $0.3 \mathrm{ml} \mathrm{s}^{-1}$. In an identical manner regarding adsorption times and washing parameters other polymers were applied on the PEI-coated gold surface. A final polyelectrolyte concentrations in the adsorption medium was $1 \mathrm{mg} \mathrm{ml}^{-1}$ in case of PSS/PAH and $0.1 \mathrm{mg} \mathrm{ml}^{-1}$ for CMC/CHI system. The adsorption and rinsing cycles were repeated until 9 double layers of particular polymer arrangements were received. All ellipsometric experiments were performed at least in duplicate.

\subsection{Electrochemistry}

All electrochemical measurements were performed using a threeelectrode BAS CV-50W Voltammetric Analyzer (BAS, Bioanalytical Systems, West Lafayette, IN, USA) with $\mathrm{Ag}|\mathrm{AgCl}| \mathrm{KCl}_{\text {sat }}$ reference electrode (BAS), a platinum wire counter electrode, and gold working electrode (MF-2014, BAS). Working electrode was double cleaned by polishing on Microcloth ${ }^{\circledR}$ PSA cloth (Buehler $\mathrm{GmbH}$, Düsseldorf, Germany) with AP-D $0.1 \mu \mathrm{m}$ Alumina suspension (Struers, Copenhagen, Denmark), followed by rinsing with water and sonication for $5 \mathrm{~min}$ in between. Then, the gold electrode was cleaned electrochemically, by means of cyclic voltammetry, for $30 \mathrm{~min}$ in $500 \mathrm{mM}$ $\mathrm{H}_{2} \mathrm{SO}_{4}$ at a scan rate of $100 \mathrm{mV} \mathrm{s}^{-1}$. Modification of the electrodes with polymers was performed by immersing for $5 \mathrm{~min}$ into $10 \mathrm{ml}$ rigorously stirred polyelectrolyte or rinsing solutions, respectively. Each time, the washing step included three freshly prepared rinsing solutions for efficient washing of loosely bound polymers. A precursor layer of PEI ( $1 \mathrm{mg} \mathrm{ml}^{-1}$ ) was, always first, adsorbed on the cleaned electrode surface either from the buffer or the aqueous solution depending on the system under the investigation. Subsequently, in the case of PSS/ $\mathrm{PAH}$ multilayers, the electrode was modified in $1 \mathrm{mg} \mathrm{ml}^{-1} \mathrm{PSS}$, washed, modified with $1 \mathrm{mg} \mathrm{ml}^{-1} \mathrm{PAH}$, and again washed. Afterwards, the procedure was repeated until required 9 bilayers were deposited. Exactly the same routine was applied for $\mathrm{CMC} / \mathrm{CHI}$ multilayer buildup though a lower concentration of polymers $\left(0.1 \mathrm{mg} \mathrm{ml}^{-1}\right)$ was used. The electrochemical measurement, i.e. cyclic voltammetry and chronoamperometry, were carried out after modification with PEI layer and later after deposition of 3, 6 and 9 polyelectrolyte bilayers under aerobic conditions at room temperature in a one-compartment $10 \mathrm{ml}$ electrochemical cell. Cyclic voltammograms of $4 \mathrm{mM}$ potassium ferricyanide solutions were performed by sweeping the potential at $100 \mathrm{mV} \mathrm{s}^{-1}$ scan rate in the range -200 to $650 \mathrm{mV}$ vs. $\mathrm{Ag}|\mathrm{AgCl}| \mathrm{KCl}_{\text {sat }}$. Chronoamperometric current responses were recorded both in the presences and in the absences of electroactive species in the supporting buffer solution by stepping potential for $10 \mathrm{~s}$ from 400 to $0 \mathrm{mV}$ vs. $\mathrm{Ag}|\mathrm{AgCl}| \mathrm{KCl}_{\text {sat }}$.

\section{Results and discussions}

\subsection{Polyelectrolyte multilayer formation}

The initial layer of PEI was applied onto a bare gold electrode surface, serving as an anchor for subsequent polyelectrolytes adsorp- tion [55]. Poly(ethylene imine) is a weak polybase, commonly used as an adhesion promoter in order to achieve a positively charged substrate with an even coating, and to minimise an influence of the surface on the growth of consecutive layers [56]. The next step in the multilayer film construction included the exposure of the electrode surface to a negatively charged polymer, either PSS or CMC, followed by a careful rinsing. Then, a positively charged polyelectrolyte, PAH or $\mathrm{CHI}$, respectively, was adsorbed and the surface washed again. The procedure was repeated until desired number of polyelectrolyte bilayers were obtained, originating in the PSS/PAH or the $\mathrm{CMC} / \mathrm{CHI}$ LbL assembled film, respectively. It should be noted that the PSS/PAH films were composed of a strong polyacid, poly(styrene sulfonate) [57] and a weak polybase, poly(allylamine hydrochloride) [32], whereas, $\mathrm{CMC} / \mathrm{CHI}$ systems were built based on a weak polyacid: carboxymethyl cellulose [58] and a weak polybase:chitosan [59].

The multilayer formation processes were carried out from two different solutions, i.e. buffer and water, which were also used in the washing steps. This yields three different cases for each system: First, the polyelectrolytes, including PEI, were dissolved and LbL assembled in buffer (composition described in Section 2.1.), for PSS/PAH film in Tris and $\mathrm{CMC} / \mathrm{CHI}$ in phosphate, and then rinsed between LbL adsorption steps with water (called the buffer-water case). The second category involved above mentioned buffers as solvents and washing media (i.e., the buffer-buffer case). The third case corresponded to the procedure in which water was the environment for both multilayer buildup and rinsing (i.e., the water-water case).

The development of the multilayer films, in terms of the amounts and the thicknesses of the layers is presented in Fig. 1 as a summary of the data from the ellipsometric monitoring. A monotonous multilayer growth can be observed for PSS/PAH polyelectrolyte system from all LbL deposition cases, whereas CHI/CMC multilayer formation can be noticed only in the buffer-water case (Fig. 1b).

Concluding on PSS/PAH polyelectrolyte system the major trend is obvious, i.e., the resulting layers from polyelectrolytes dissolved in water are much thinner than those adsorbed from the buffer. The thickest layers of PSS/PAH were obtained when polymers had been dissolved in buffer and washed with water in between (i.e., the bufferwater case, Fig. 1a). After the initial slow growth of first bilayers, the buildup of the film was linear with the average of the thickness increase of approximately $2 \mathrm{~nm}$ per polyelectrolyte bilayer. In the same manner, the increment of adsorbed amount followed linear multilayer development, with about $1.92 \mathrm{mg} \mathrm{m}^{-2}$ increase per polyelectrolyte bilayer. These characteristics resulted into $0.94 \mathrm{~g} \mathrm{~cm}^{-3}$ polyelectrolyte density in the film at the end of the entire experiment. The bufferbuffer and the water-water cases revealed a linear PSS/PAH growth in respect to the adsorbed amounts and the thicknesses (Fig. 1c and e). The multilayer growth parameters in terms of the adsorbed amount, the thickness growth per bilayer and the final density of polyelectrolytes in the film were $1.71 \mathrm{mg} \mathrm{m}^{-2}$ vs. $0.56 \mathrm{mg} \mathrm{m}^{-2}, 1.50 \mathrm{~nm}$ vs. $0.80 \mathrm{~nm}$, and $1.14 \mathrm{~g} \mathrm{~cm}^{-3}$ vs. $0.72 \mathrm{~g} \mathrm{~cm}^{-3}$, for the buffer-buffer vs. water-water cases, respectively.

Consider the electrostatic forces in the present system the electrostatic repulsion between polymer segments of the same charge prevails in the water-water case (Fig. 1e) and induces stretching out of the polyelectrolyte chains, which results in to an extended, rodshaped polymer conformation. Therefore, the polymers form a flat LbL film upon adsorption. The lowest density of the film $\left(0.72 \mathrm{~g} \mathrm{~cm}^{-3}\right)$ in this case indicates that the rod-like structure does not allow ordered packing of polymer molecules into a dense film.

At higher ionic strength, the long range electrostatic forces are nearly fully screened and thus the repulsive forces between the charged segments along the polymer chains are minimised and the polymers acquire random coil conformations. Consequently, deposition of the PSS/PAH from buffer solution (Fig. 1c) leads to higher amounts of the polyelectrolytes and larger thicknesses of the deposited layers. The minimised repulsion between the random coil 

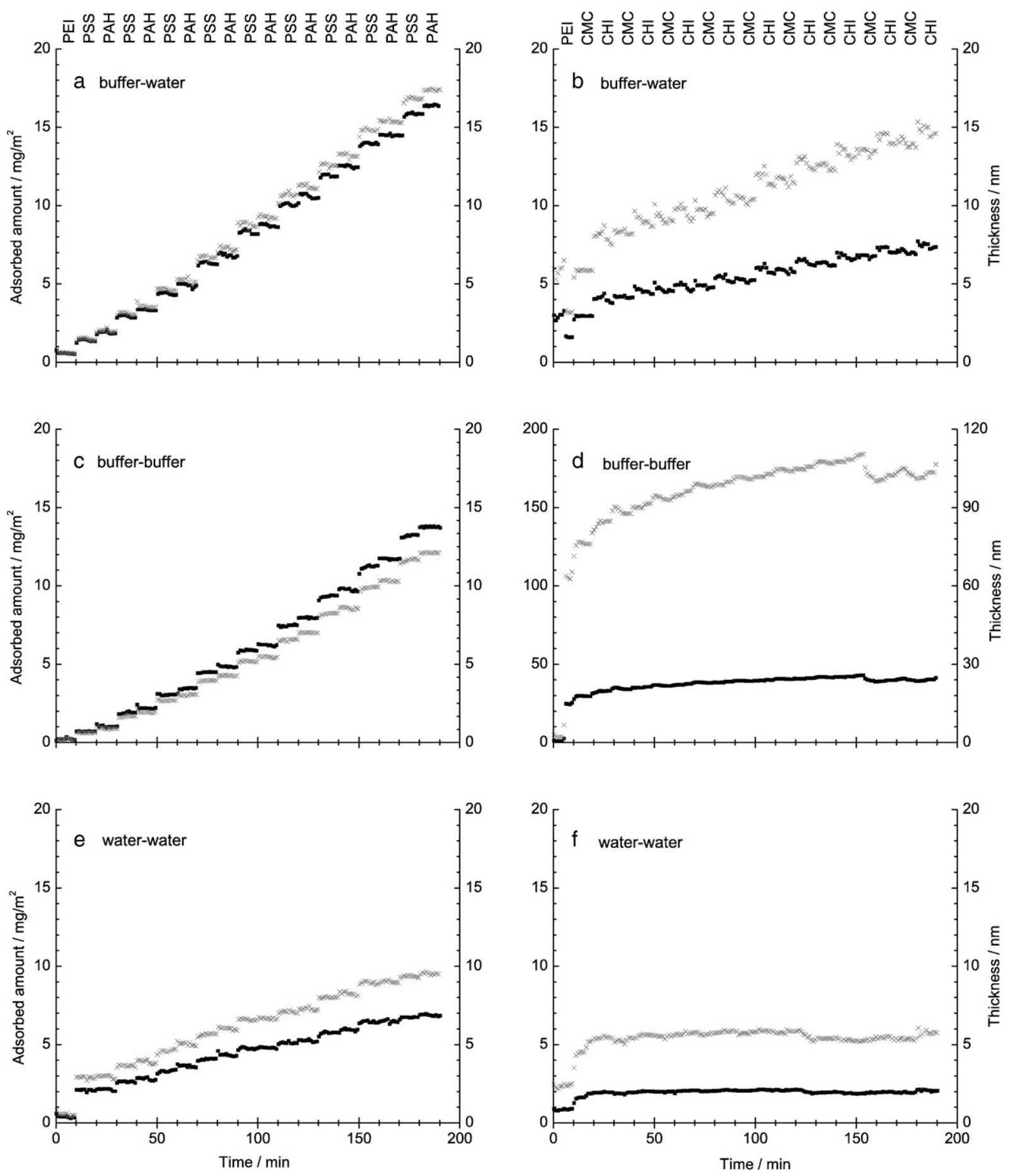

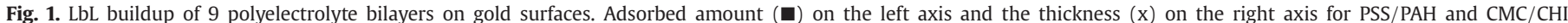
polyelectrolyte systems. (a) and (b) show the buffer-water case; (c) and (d) describe the buffer-buffer case; (e) and (f) illustrate the water-water case.

of the polymers results in the most dense polyelectrolyte films $(1.14 \mathrm{~g}$ $\mathrm{cm}^{-3}$ ) if compared among the three studied cases.

The highest growth of the PSS/PAH films in terms of mass and thickness resulting into intermediate film density $\left(0.94 \mathrm{~g} \mathrm{~cm}^{-3}\right)$ at the conditions when washing is done by pure water (i.e., the buffer-water case) (Fig. 1a) can also be rationalised. During the washing step with water, counter ions present in multilayers might diffuse out of the layers into water, causing higher charges unbalance in the bulk film. As a consequence the uncompensated charges within the film drive a thicker adsorption of the subsequent polyelectrolyte layer, and overall thicker film is formed. The washing with water should also increase the repulsion between the polymer coils in the film and as a consequence reduce the overall density of the PSS/PAH multilayer. Above explanation can be supported by the previous studies of PSS/ PAH films built in the presence of salt. Ladam et al. [34] showed that LbL multilayers were affected by diminishing the ionic strength after the buildup was completed, resulting in significant swelling (i.e., about 20\%).

The CMC/CHI polysaccharide films adsorbed on gold surfaces behave considerably different if compared to PSS/PAH multilayers. The growth of thicknesses and adsorbed amounts of $\mathrm{CMC} / \mathrm{CHI}$ can only be seen in two cases, namely the buffer-water (Fig. 1b) and the 
buffer-buffer (Fig. 1d) cases, respectively. The growth is very excessive for the first bilayers and becomes linear for subsequent layers with a thickness increase of $0.90 \mathrm{~nm}$ and $3.50 \mathrm{~nm}$ per bilayer in the bufferwater and the buffer-buffer case, respectively. These values are comparable to the PSS/PAH multilayer formation. In the linear growth phase, the adsorbed amount per $\mathrm{CMC} / \mathrm{CHI}$ polyelectrolyte bilayer is, however, considerably different with $0.46 \mathrm{mg} \mathrm{m}^{-2}$ and $1.37 \mathrm{mg} \mathrm{m}^{-2}$ per bilayer for the buffer-water and the buffer-buffer case, respectively. The mass growth and the final polyelectrolyte densities of $\mathrm{CMC} / \mathrm{CHI}$ films $\left(0.50 \mathrm{~g} \mathrm{~cm}^{-3}\right.$ for the buffer-water and $0.39 \mathrm{~g} \mathrm{~cm}^{-3}$ for the buffer-buffer case) are much lower than the same characteristics of the PSS/PAH polyelectrolyte films described above.

A high amount of $\mathrm{CMC} / \mathrm{CHI}$ polyelectrolytes adsorbed in the first bilayer suggests that the charge and the amount of surface confined PEI play a significant role. This is specifically true when polyethyleneimine is adsorbed from the buffer solution and the surface is washed with the high ionic strength buffer (Fig. 1d). Most probably, great amount of random coil PEI is left on the surface. This provides a considerable positive charge associated with the surface and as anticipated, such a surface will adsorb considerable amount of CMC/ $\mathrm{CHI}$ during the first bilayer formation. If, however, PEI-treated surface is washed with water (Fig. 1b), it is obvious that adsorbed PEI will swell and some amount of PEI will desorb due to intermolecular charge repulsion. Hence, washing PEI-treated surface with water reduces total charge adsorbed on the surface and thus results in a lower amount of $\mathrm{CMC} / \mathrm{CHI}$ adsorbed with the first bilayer if compared with the buffer washing case.

In the water-water case the growth of the $\mathrm{CMC} / \mathrm{CHI}$ film stops already after the first bilayer (Fig. 1f). The thickness of the multilayer

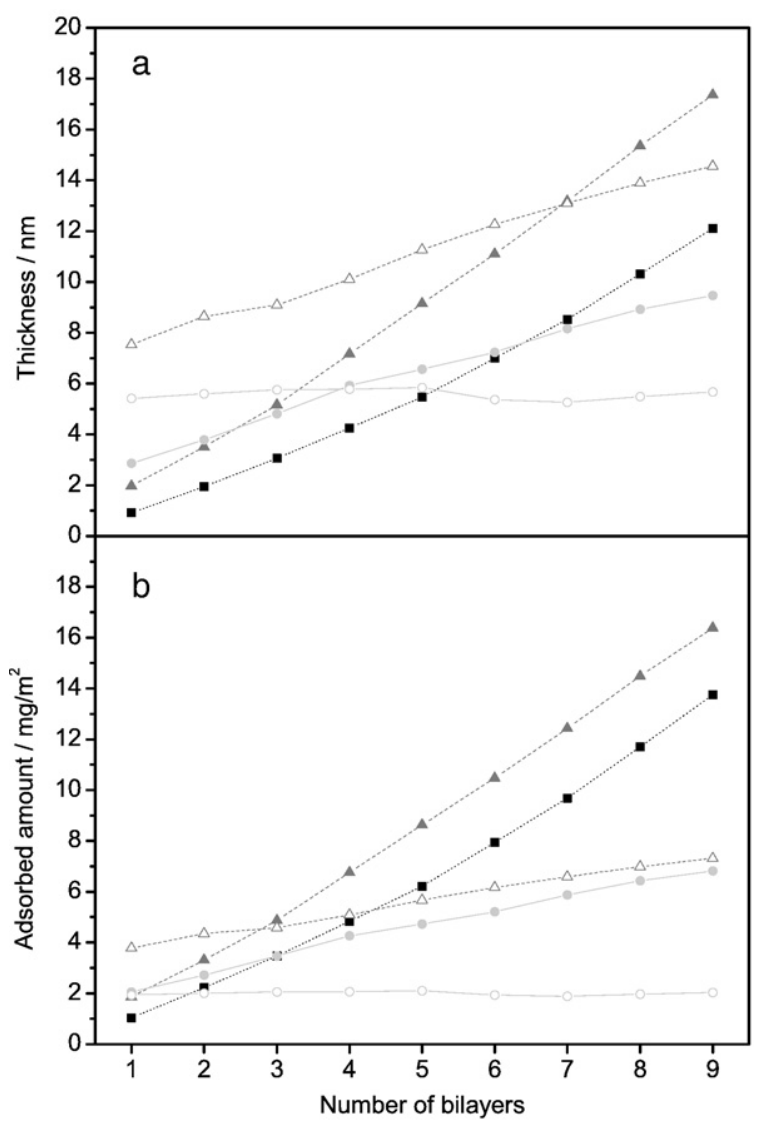

Fig. 2. Progression of thickness (a) and adsorbed amount (b) vs. number of adsorbed bilayers during the buildup of the PSS/PAH multilayers for the buffer-buffer case ( $\mathbf{\square})$, the buffer-water case $(\boldsymbol{\Delta})$, and the water-water case $(\odot)$, likewise the development of the $\mathrm{CMC} / \mathrm{CHI}$ system for the buffer-water case $(\Delta)$ and the water-water case $(O)$. films is strongly affected by the degree of charge density on the polymer. A minimum amount of charge density on polyelectrolyte chain is needed for the formation of a stable multilayer. The distribution of the charge density along the chains plays also an important role, since it was shown that short, strongly charged blocks were sufficient to construct multilayers [60]. Therefore, it could be concluded that $\mathrm{CMC}$ and $\mathrm{CHI}$ do not retain sufficient charges to assemble the multilayer in water-water system. More generally, a multilayer assembly from the weak polyelectrolyte (polybase/polyacid) couple requires much better control over $\mathrm{pH}$ of the solution.

From Fig. 2, which summarises nearly all cases of multilayer formation procedures described in Fig. 1, it can be seen that, the PSS/ $\mathrm{PAH}$ polyelectrolyte system exhibits an approximately linear growth of multilayers for each LbL assembly condition/case studied. Generally, the $\mathrm{CMC} / \mathrm{CHI}$ also shows linear LbL assembly. However, the formation of the first bilayer is an exception. Due to a relatively high PEI charge at the PEI-treated surface a high amount of weak CMC/CHI polyelectrolytes is adsorbed during the initial growth of the LbL film. Additionally, in the case of weak polyelectrolytes, the $\mathrm{pH}$ control in water is not sufficient to realise the buildup of the multilayers. The mass density of the PSS/PAH multilayers (range $0.72-1.14 \mathrm{~g} \mathrm{~cm}^{-3}$ ) corresponds to the values obtained by Lösche et al. [21] for the same assemblies adsorbed on silica surfaces: 0.81 and $1.13 \mathrm{~g} \mathrm{~cm}^{-3}$ as fully hydrated and fully dehydrated films, respectively, and interestingly, close to the density of protein films $\left(1.33 \mathrm{~g} \mathrm{~cm}^{-3}\right)$ [61] or protein bilayers at the gold supports, e.g., composed of lactoperoxidase adsorbed on bovine serum albumin $\left(1.25 \mathrm{~g} \mathrm{~cm}^{-3}\right)$ [48]. The densities of $\mathrm{CMC} / \mathrm{CHI}$ polyelectrolytes (range $0.39-0.50 \mathrm{~g} \mathrm{~cm}^{-3}$ ) can be compared to previously studied films of extensively glucosylated proteins, e.g., human salivary mucin layer on bare gold [48] or hydrophobized silica surfaces [62] which have the density of approximately $0.12 \mathrm{~g} \mathrm{~cm}^{-3}$ or bovine mucin film with an order of magnitude lower density of $0.01-0.05 \mathrm{~g} \mathrm{~cm}^{-3}[48,63]$.

\subsection{Electrochemical studies of the assembled multilayers}

The multilayers evaluated by using ellipsometry were simultaneously studied with electrochemical methods. The aim was to assess the layer permeability to small molecules, in our particular case [Fe $\left.(\mathrm{CN})_{6}\right]^{3-/ 4-}$ ion, i.e., ferri-/ferrocyanide ion. The permeability of ferri-/ ferrocyanide through the multilayers can be easily measured as an electrical current, which results from the oxidation or reduction of the redox molecules at the electrode surface. Obviously, the current depends on the transport of electroactive species through the polymer layer, which mechanistically can be imagined as a diffusion process of ferri-/ ferrocyanide through the pores/pinholes in a polyelectrolyte layer or through homogeneous polymeric film matrix.

In our experiments, first, the film permeability to the redox ions was studied by cyclic voltammetry. The measurements were performed with polyelectrolyte modified gold electrodes, specifically, after PEI, and additionally, after 3, 6, and 9 polyelectrolyte bilayers deposited on the electrode surface. Fig. 3 summarises cyclic voltammograms obtained with modified gold electrodes at different conditions. At first, cyclic voltammetry enables to compare quickly and qualitatively the polymer multilayer coatings by analyzing the value of the peak current and the shapes of the voltammograms. As it can be seen from Fig. 3, only the PSS/PAH LbL buildup using buffer deposition step and water (Fig. 3a) or buffer (Fig. 3c) washing step result into well pronounced diminish of the peak currents. The process is obviously related to the blocking of the electrode surface by the insulating polymer layers. It is also easy to note that cyclic voltammograms (CVs) turn from peak-shaped to sigmoid-shaped as a number of PSS/PAH bilayers increases. This indicates that the diffusion of ferri-/ ferrocyanide through micropores or pinholes in the polyelectrolyte layer determines the electrode current. From potential scan rate, $\nu$, at which the shape of CVs become sigmoidal we can 

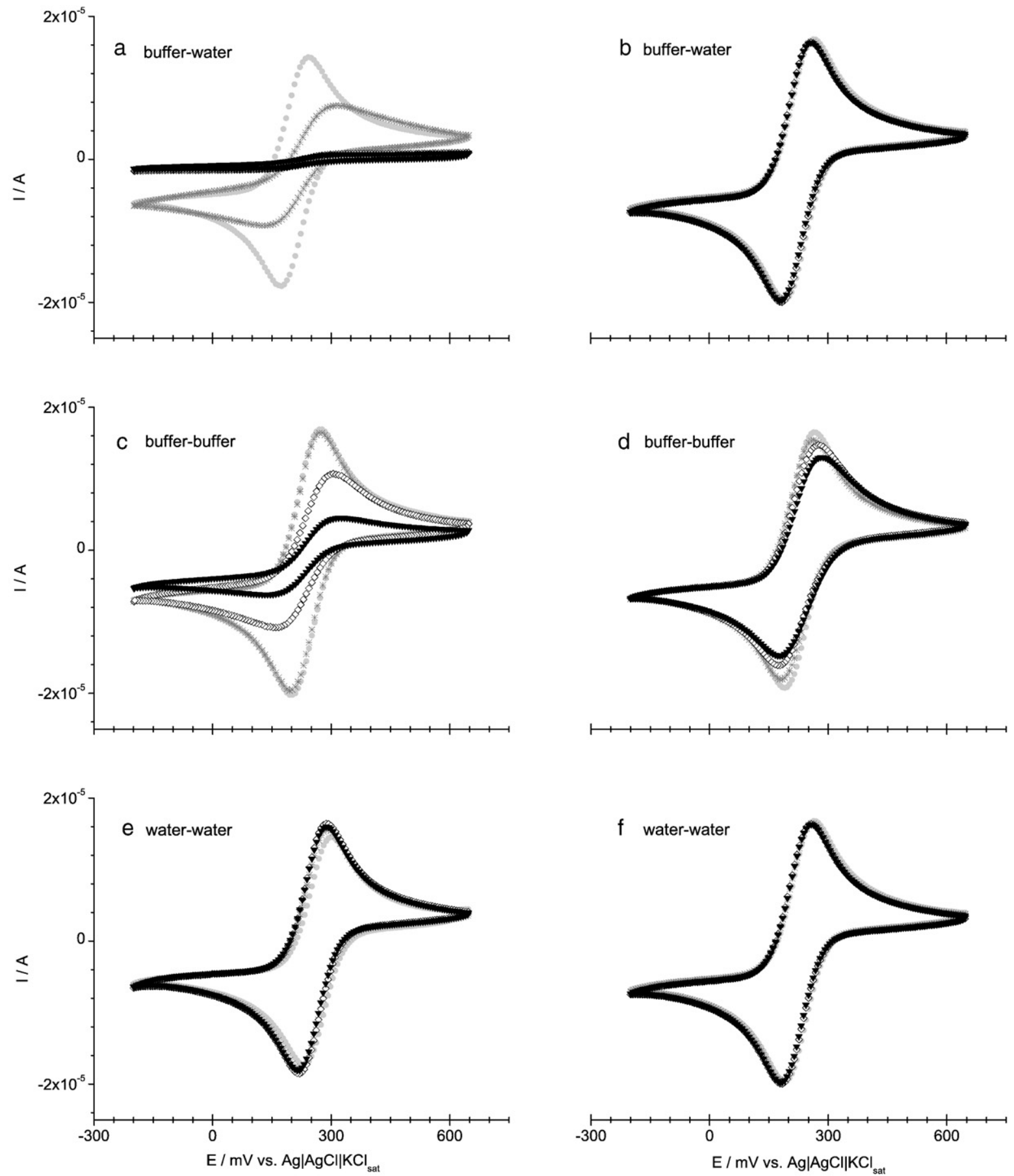

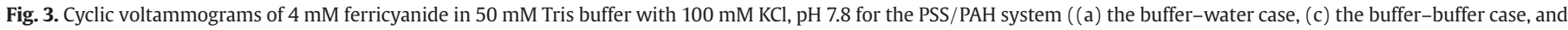

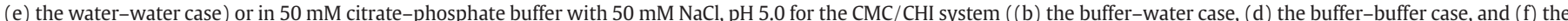

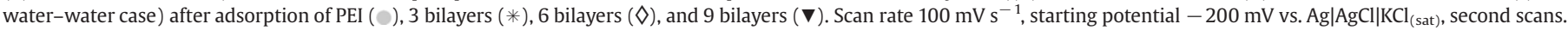

estimate an approximate radius of the micropores, $r_{0}\left(\nu \approx R T D / n F r_{0}\right.$, where $R$ is molar gas constant, $T$ is absolute temperature, $D$ is diffusion coefficient for redox couple, $7.8 * 10^{-6} \mathrm{~cm}^{2} \mathrm{~s}^{-1}, n=1$ is a number of electrons in electrochemical reaction, and $F$ is Faraday constant). Taking $\nu=100 \mathrm{mV} \mathrm{s}^{-1}$ the calculation returns a $14 \mu \mathrm{m}$ micropore radius.

The absence of a noticeable changes in the CVs recorded with the electrodes modified at other conditions (Fig. 3b, d, e, and f) indicates that the polyelectrolyte layers on these electrodes have much bigger pores and even might form relatively homogeneous, low density, gellike film structures. The comparison of CVs (Fig. 3) and ellipsometry data (Fig. 1) allows us to conclude that the layers with polyelectrolyte density below $0.80 \mathrm{~g} \mathrm{~cm}^{-3}$ do not restrict the diffusion of the redox couple (ferri-/ferrocyanide in this case) to the electrode surface at relatively slow potential sweep rates, i.e., cyclic voltammetry measurements at $100 \mathrm{mV} \mathrm{s}^{-1}$. The absence of a pronounced effect on the shape of the CVs with the number of growing layers probably can be expected for diminutive growing LbL polyelectrolyte 

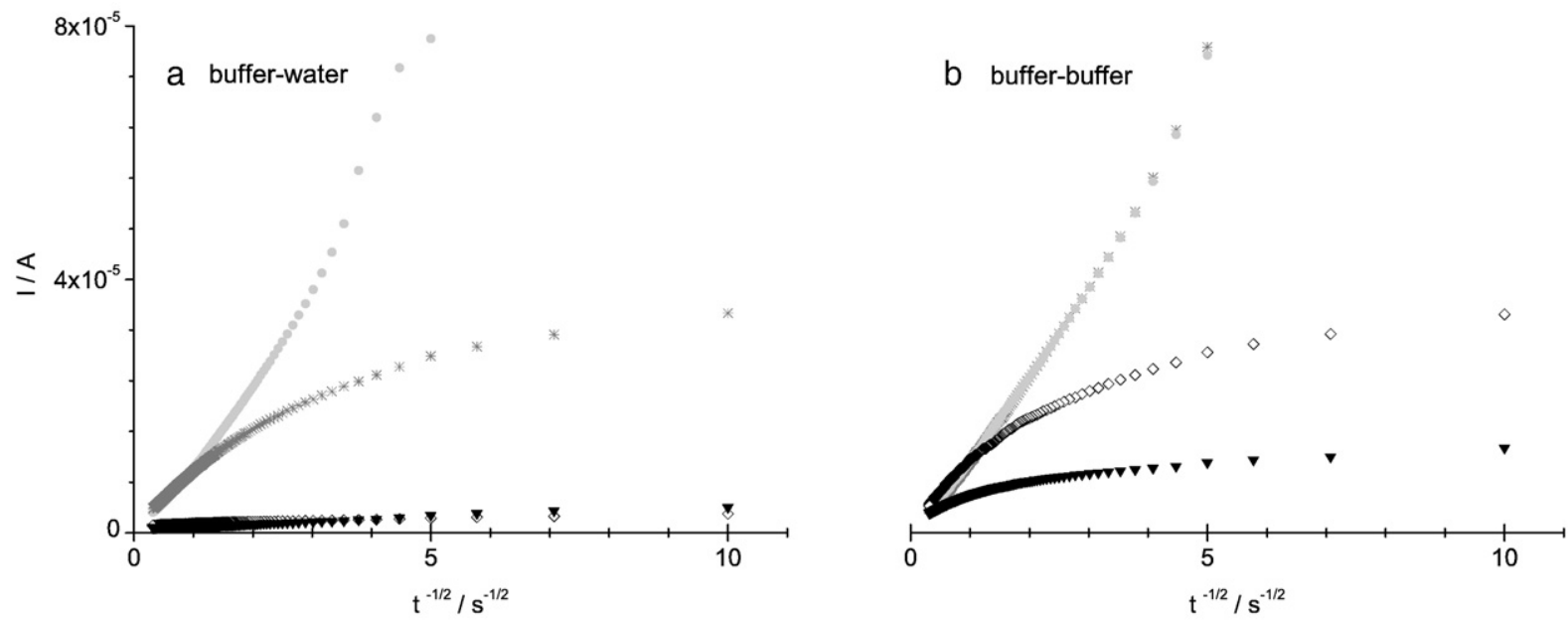

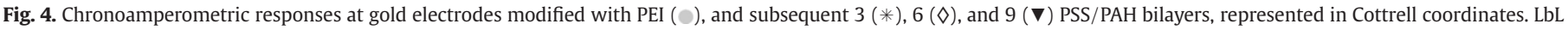

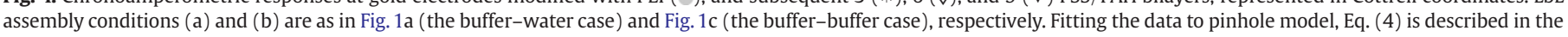
text and the parameters are provided in the table.

assemblies (compare Figs. 2 and 3). It is logical that the LbL assembly of such multilayer films is driven by weaker charge interactions, which in return gives a loose layer structure.

To characterise the structure of the LbL films more comprehensively we additionally explored another electrochemical technique, namely chronoamperometry. Chronoamperometric measurements return a current-time dependency as a response to a potential step at the electrode. The chronoamperometric current is mathematically described by modelling the diffusion of the redox species through (i) a membrane, i.e., homogeneous polymer layers at the electrode surface or through (ii) pinholes in the impermeable film, i.e., the holes in the polymer film. At a bare and cleaned electrode chronoamperometric response follows the Cottrell equation (Eq. (2)), which turns into different expressions depending whether polymer layers on the electrode behave as a homogeneous membrane (Eq. (3)) or as a layer with holes (Eq. (4)):

$i_{c}(t)=\frac{n F A_{\text {geom }} C \sqrt{D}}{\sqrt{\pi t}}$

where $A_{\text {geom }}$ is a geometric area of the electrode, $t$ is a time (in our case from 0 to $10 \mathrm{~s}$ ) and $C$ is the concentration of the redox species in solution (in our case $C=4 \mathrm{mM}$ of ferricyanide). Other constants have already been defined above by considering CVs. For a membrane model the current has the following expression [44]:

$i(t)=i_{c}(\tau) \frac{K}{\gamma}\left\{1+2 \sum\left[\frac{1-\frac{K}{\gamma}}{1+\frac{K}{\gamma}}\right]^{j} \exp \left(\frac{-j^{2}}{\tau}\right)\right\}$,

$K=\frac{\left.c_{\mathrm{m}}(\delta, t)\right)}{c_{\mathrm{sol}}(\delta, t)}, \gamma=\sqrt{\frac{D_{\mathrm{sol}}}{D_{\mathrm{m}}}}, \tau=\frac{D_{\mathrm{m}} t}{\delta^{2}}$

where $c_{\mathrm{m}}, D_{\mathrm{m}}, c_{\mathrm{sol}}$, and $D_{\text {sol }}$ are concentrations and diffusion coefficients of redox species in membrane and solution, respectively, $K$ is an extraction coefficient, and $\delta=\sqrt{\pi \cdot D_{\text {sol }} \cdot t}$. For a pinhole model a chronoamperometric current response is [64,65]:

$i(t)=\frac{n F A_{\text {geom }} C \sqrt{D}}{\sqrt{\pi t}}\left\{1-\exp \left[\frac{-\sqrt{\pi D t}}{2}\left(N 2 \pi r_{0}\right)-\left(N \pi r_{0}^{2}\right)\right]\right\}$

where $N$ is a number pinholes per $\mathrm{cm}^{2}$ in the membrane and $r_{0}$ is the most representative radius of the pinhole.
All LbL-modified electrodes were subjected to chronoamperometric measurements with potential pulse for $10 \mathrm{~s}$. However, as in a case with CV measurements, only two of LbL structures deposited on electrodes showed currents lower than the diffusion limited current at unmodified electrodes. Specifically, these were LbL structures of PSS/ $\mathrm{PAH}$ polyelectrolytes deposited from buffer and washed with buffer or water in between the deposition steps. The chronoamperometric responses of these electrodes as current vs. time dependence (Fig. 4) were fitted into membrane and pinhole models, respectively (Eqs. (3) and (4)). It appeared that reasonable fitting was possible only to the pinhole model, which indicates that diffusion limiting LbL structures with up to 9 bilayers do not represent homogeneous membranes and, thus can be considered as rather inhomogeneous and pinhole dominating polyelectrolyte layers.

Fitting chronoamperometric responses to the pinhole model revealed interesting features. For both PSS/PAH LbL deposition conditions, i.e., the buffer-buffer and the buffer-water cases, the chronoamperometric response is apparently dominated by the diffusion of the redox species through the pinholes with a radius between 8 and $16 \mu \mathrm{m}$. This pinhole radius apparently determines the chronoamperometric current response even when further building the LbL structure from 3 to 9 PSS/PAH polyelectrolyte bilayers. The calculation actually shows that the growth in the polyelectrolyte

Table 1

Characteristics calculated from fitting the chronoamperometric current responses to pinhole model, Eq. (4), where $A_{\text {geom }}$ is the geometric area of the electrode, $N$ is the number of microelectrodes per $\mathrm{cm}^{2}$ and $r_{0}$ is the radius of microelectrode.

\begin{tabular}{lccc}
\hline & $A_{\text {geom }} / \mathrm{cm}^{2}$ & $N$ & $r_{0} / \mu \mathrm{m}$ \\
\hline The PSS/PAH LbL deposited from & buffer and washed with & buffer in between the deposition steps \\
Bare electrode & 0.02 & $\mathrm{ND}$ & $\mathrm{ND}$ \\
After PEI & 0.02 & $\mathrm{ND}$ & $\mathrm{ND}$ \\
After 3 bilayers & 0.02 & $\mathrm{ND}$ & $\mathrm{ND}$ \\
After 6 bilayers & 0.023 & 110000 & 8.6 \\
After 9 bilayers & 0.018 & 48300 & 8.3 \\
& & & \\
The PSS/PAH LbL deposited from buffer and washed with water in between the deposition steps \\
Bare electrode & 0.02 & $\mathrm{ND}$ & $\mathrm{ND}$ \\
After PEI & 0.02 & $\mathrm{ND}$ & $\mathrm{ND}$ \\
After 3 bilayers & 0.02 & 140000 & 8.8 \\
After 6 bilayers & 0.02 & 9000 & 8.8 \\
After 9 bilayers & 0.02 & 300 & 16 \\
\hline
\end{tabular}

ND (no data) denotes that the parameter has no meaning since the chronoamperometric response is determined by the diffusion of ferri-/ferrocyanide to the entire area of the electrode and thus the chronoamperometric response is described by Cottrell equation, Eq. (2). 


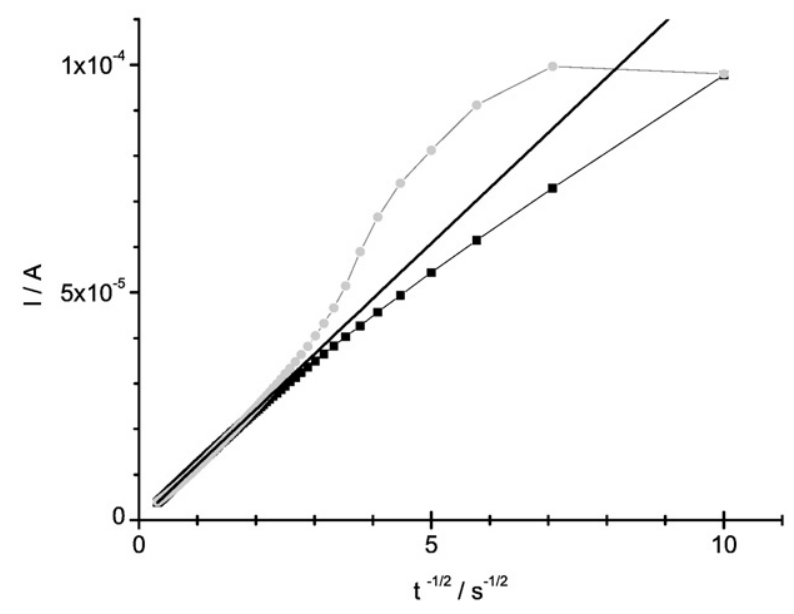

Fig. 5. An average chronoamperometric response in Cottrell coordinates at PEI modified gold electrodes (๑). Theoretically simulated diffusion limited response (Eq. (2)) at bare gold electrodes (solid line). Theoretical prediction of partially blocked electrode ( $\mathbf{\square}$ ) was calculated applying Eq. (4).

bilayers on the surface of the electrodes systematically reduces the number of the pinholes. This is obvious from the calculation results summarised in Table 1.

Another important feature could be noticed from the chronoamperometric measurements, namely that at all PEI or polyelectrolyte modified electrodes, without the pinhole structure detected, the measured current is higher than the diffusion limited current. As an example, Fig. 5 depicts an average of chronoamperometric response at PEI modified electrodes.

The measured chronoamperometric responses higher than the current limited by the diffusion can be explained by noticing that all chronoamperometric measurements were made with polyelectrolyte modified electrodes where the last deposited polyelectrolyte was positively charged, i.e., PEI, PAH or CHI. A positively charged polyelectrolyte obviously attracts negatively charged ferri-/ferrocyanide. We attempted to estimate an amount of pre-concentrated redox molecules for PEI modified electrodes, because at these electrodes we have observed the highest deviation from the diffusion limited current. The estimation was done by integrating the current which was above the diffusion limit at the partially blocked electrode, i.e., the difference between the data represented with circles and square symbols in Fig. 5. The mean value of pre-concentrated redox charge

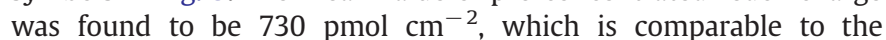
concentration of a fully packed thiol monolayer at gold electrodes (750-850 pmol cm ${ }^{-2}$ [66]). From the time period when this higher current is observed we also can estimate that the preconcentration extends to approximately $10 \mu \mathrm{m}$ and thus would result in about $20 \%$ higher adjacent to the surface concentration of redox molecules than in solution. This estimate was calculated by using diffusion coefficient of the ferri-/ ferrocyanide in solution. Unfortunately, $10 \mu \mathrm{m}$ distance of disturbed concentration is probably not realistic if considering the distances that charge-charge interactions can extent in $100 \mathrm{mM} \mathrm{KCl}$ electrolyte. The overestimation is obviously due to not accounted charge interactions between the positively charged PEI and ferri-/ ferrocyanide, which disturb the diffusion of the redox molecules at the electrodes. The amount of pre-concentrated redox molecules though might be realistic.

\section{Conclusions}

Combination of ellipsometry and electrochemistry was used to characterise LbL deposited polyelectrolyte structures on gold surfaces initially pre-treated with PEI. Two polyelectrolyte systems and three different LbL deposition condition were compared. It was found that the polyelectrolyte system consisting of strong acids (i.e., PSS) and weak basis (i.e., PAH) result into relatively dense surface confined LbL polyelectrolyte structures. The polyelectrolyte system of weak polysaccharides (i.e., CMC and $\mathrm{CHI}$ ) yielded very low density of LbL films with estimated polyelectrolyte density in the film as low as $0.39 \mathrm{~g} \mathrm{~cm}^{-3}$. This density is much lower than a density of globular protein films and a little bit higher that the density of surface confined mucin layers, which are known to be highly hydrated.

Electrochemical measurements of diffusion properties of small redox molecules through the polyelectrolyte films confirmed that only PSS/PAH polyelectrolyte films restricts permeability of small molecules. This feature was well pronounced when LbL PSS/PAH film was assembled from buffered solution and washed in between LbL steps with buffer or water. Therefore compact polyelectrolyte films can be envisaged as membranes containing a high number of micrometer pinholes. During the deposition process, i.e., by increasing the number of polyelectrolyte bilayers, the number of pinholes quickly reduces. The data, however, indicate that the diameter of pinholes, which determines the main pathway of diffusion of the small redox molecules through the films might be of 10th $\mu \mathrm{m}$ in radius and can stay the same at the initial polyelectrolyte LbL assembling steps (in our case up to 9 polyelectrolyte bilayers).

\section{Acknowledgements}

The work was financially supported by The Marie Curie "BIONEL" project (ref. no.: 373-4515/2004), The Swedish Research Council (project 621-2005-3581), The European Commission (project FP7NMP-2008-229255), and Malmö University. Authors are thankful to Dr. Christina Bjerkén at Malmö University and Dr. Ulla Elofsson at the Institute for Surface Chemistry, Stockholm, for contribution at different stages of this project.

\section{References}

[1] W. Zhao, J.-J. Xu, H.-Y. Chen, Electrochemical biosensors based on layer-by-layer assemblies, Electroanalysis 18 (2006) 1737-1748.

[2] P.K.H. Ho, J.-S. Kim, J.H. Burroughes, H. Becker, F.Y.L. Sam, T.M. Brown, F. Cacialli, R.H. Friend, Molecular-scale interface engineering for polymer light-emitting diodes, Nature (London) 404 (2000) 481-484

[3] F. Caruso, R.A. Caruso, H. Moehwald, Nanoengineering of inorganic and hybrid hollow spheres by colloidal templating, Science (Washington, D. C.) 282 (1998) 1111-1114.

[4] S.C. Olugebefola, W.A. Kuhlman, M.F. Rubner, A.M. Mayes, Photopatterned nanoporosity in polyelectrolyte multilayer films, Langmuir 24 (2008) 5172-5178.

[5] M. Nolte, A. Fery, Freestanding polyelectrolyte multilayers as functional and construction elements, IEE Proc.: Nanobiotechnol. 153 (2006) 112-120.

[6] C.S. Peyratout, L. Daehne, Tailor-made polyelectrolyte microcapsules: from multilayers to smart containers, Angew. Chem., Int. Ed. 43 (2004) 3762-3783.

[7] G. Decher, J.D. Hong, J. Schmitt, Buildup of ultrathin multilayer films by a selfassembly process: III. Consecutively alternating adsorption of anionic and cationic polyelectrolytes on charged surfaces, Thin Solid Films 210-211 (1992) 831-835.

[8] R. Dronov, D.G. Kurth, H. Moehwald, F.W. Scheller, F. Lisdat, Communication in a protein stack: electron transfer between cytochrome $c$ and bilirubin oxidase within a polyelectrolyte multilayer, Angew. Chem., Int. Ed. 47 (2008) 3000-3003.

[9] N.G. Balabushevitch, G.B. Sukhorukov, N.A. Moroz, D.V. Volodkin, N.I. Larionova, E. Donath, H. Mohwald, Encapsulation of proteins by layer-by-layer adsorption of polyelectrolytes onto protein aggregates: factors regulating the protein release, Biotechnol. Bioeng. 76 (2001) 207-213.

[10] G.B. Sukhorukov, E. Donath, S. Davis, H. Lichtenfeld, F. Caruso, V.I. Popov, H. Mohwald, Stepwise polyelectrolyte assembly on particle surfaces: a novel approach to colloid design, Polym. Adv. Technol. 9 (1998) 759-767.

[11] G. Schneider, G. Decher, Functional core/shell nanoparticles via layer-by-layer assembly. Investigation of the experimental parameters for controlling particle aggregation and for enhancing dispersion stability, Langmuir 24 (2008) 1778-1789.

[12] A.S. Hoffman, Immobilization of biomolecules and cells on and within polymeric biomaterials, Clin. Mater. 11 (1992) 61-65.

[13] A.J. Khopade, F. Caruso, Investigation of the factors influencing the formation of dendrimer/polyanion multilayer films, Langmuir 18 (2002) 7669-7676.

[14] S. Kidambi, J. Dai, J. Li, M.L. Bruening, Selective hydrogenation by Pd nanoparticles embedded in polyelectrolyte multilayers, J. Am. Chem. Soc. 126 (2004) 2658-2659.

[15] P. Bertrand, A. Jonas, A. Laschewsky, R. Legras, Ultrathin polymer coatings by complexation of polyelectrolytes at interfaces: suitable materials, structure and properties, Macromol. Rapid Commun. 21 (2000) 319-348. 
[16] U. Voigt, V. Khrenov, K. Tauer, M. Hahn, W. Jaeger, R. von Klitzing, The effect of polymer charge density and charge distribution on the formation of multilayers, J. Phys.: Condens. Matter 15 (2003) S213-S218.

[17] N.G. Hoogeveen, M.A.C. Stuart, G.J. Fleer, M.R. Boehmer, Formation and stability of multilayers of polyelectrolytes, Langmuir 12 (1996) 3675-3681.

[18] S.L. Clark, P.T. Hammond, The role of secondary interactions in selective electrostatic multilayer deposition, Langmuir 16 (2000) 10206-10214.

[19] G. Decher, Y. Lvov, J. Schmitt, Proof of multilayer structural organization in selfassembled polycation-polyanion molecular films, Thin Solid Films 244 (1994) 772-777.

[20] G. Decher, Fuzzy nanoassemblies: toward layered polymeric multicomposites, Science (Washington, D. C.) 277 (1997) 1232-1237.

[21] M. Loesche, J. Schmitt, G. Decher, W.G. Bouwman, K. Kjaer, Detailed structure of molecularly thin polyelectrolyte multilayer films on solid substrates as revealed by neutron reflectometry, Macromolecules 31 (1998) 8893-8906.

[22] J. Schmitt, T. Gruenewald, G. Decher, P.S. Pershan, K. Kjaer, M. Loesche, Internal structure of layer-by-layer adsorbed polyelectrolyte films: a neutron and X-ray reflectivity study, Macromolecules 26 (1993) 7058-7063.

[23] F. Caruso, D.N. Furlong, K. Ariga, I. Ichinose, T. Kunitake, Characterization of polyelectrolyte-protein multilayer films by atomic force microscopy, scanning electron microscopy, and Fourier transform infrared reflection-absorption spectroscopy, Langmuir 14 (1998) 4559-4565.

[24] T. Mauser, C. Dejugnat, H. Moehwald, G.B. Sukhorukov, Microcapsules made of weak polyelectrolytes: templating and stimuli-responsive properties, Langmuir 22 (2006) 5888-5893.

[25] J. Dejeu, R. Salut, M. Spajer, F. Membrey, A. Foissy, D. Charraut, Self-assembled film thickness determination by focused ion beam. Appl. Surf. Sci. 254 (2008) 5506-5510.

[26] F. Caruso, K. Niikura, D.N. Furlong, Y. Okahata, 1. Ultrathin multilayer polyelectrolyte films on gold: construction and thickness determination, Langmuir 13 (1997) 3422-3426.

[27] F. Boulmedais, V. Ball, P. Schwinte, B. Frisch, P. Schaaf, J.-C. Voegel, Buildup of exponentially growing multilayer polypeptide films with internal secondary structure, Langmuir 19 (2003) 440-445.

[28] T.J. Halthur, U.M. Elofsson, Multilayers of charged polypeptides as studied by in situ ellipsometry and quartz crystal microbalance with dissipation, Langmuir 20 (2004) 1739-1745.

[29] V. Garcia-Morales, T.H. Silva, C. Moura, J.A. Manzanares, F. Silva, Ion transport through polyelectrolyte multilayers under steady-state conditions, J. Electroanal. Chem. 569 (2004) 111-119.

[30] J.B. Schlenoff, H. Ly, M. Li, Charge and mass balance in polyelectrolyte multilayers, J. Am. Chem. Soc. 120 (1998) 7626-7634.

[31] R. Steitz, V. Leiner, R. Siebrecht, R.v. Klitzing, Influence of the ionic strength on the structure of polyelectrolyte films at the solid/liquid interface, Colloids Surf., A 163 (2000) 63-70.

[32] S.S. Shiratori, M.F. Rubner, pH-dependent thickness behavior of sequentially adsorbed layers of weak polyelectrolytes, Macromolecules 33 (2000) 4213-4219.

[33] C. Porcel, P. Lavalle, V. Ball, G. Decher, B. Senger, J.-C. Voegel, P. Schaaf, From exponential to linear growth in polyelectrolyte multilayers, Langmuir 22 (2006) 4376-4383.

[34] G. Ladam, P. Schaad, J.C. Voegel, P. Schaaf, G. Decher, F. Cuisinier, In situ determination of the structural properties of initially deposited polyelectrolyte multilayers, Langmuir 16 (2000) 1249-1255.

[35] R.A. McAloney, M. Sinyor, V. Dudnik, M.C. Goh, Atomic force microscopy studies of salt effects on polyelectrolyte multilayer film morphology, Langmuir 17 (2001) 6655-6663.

[36] C. Picart, P. Lavalle, P. Hubert, F.J.G. Cuisinier, G. Decher, P. Schaaf, J.C. Voegel, Buildup mechanism for poly(L-lysine)/hyaluronic acid films onto a solid surface, Langmuir 17 (2001) 7414-7424.

[37] P. Lavalle, C. Gergely, F.J.G. Cuisinier, G. Decher, P. Schaaf, J.C. Voegel, C. Picart Comparison of the structure of polyelectrolyte multilayer films exhibiting a linear and an exponential growth regime: an in situ atomic force microscopy study, Macromolecules 35 (2002) 4458-4465.

[38] D. Collin, P. Lavalle, J.M. Garza, J.-C. Voegel, P. Schaaf, P. Martinoty, Mechanical properties of cross-linked hyaluronic acid/poly-(L-lysine) multilayer films, Macromolecules 37 (2004) 10195-10198.

[39] P. Lavalle, V. Vivet, N. Jessel, G. Decher, J.-C. Voegel, P.J. Mesini, P. Schaaf, Direct evidence for vertical diffusion and exchange processes of polyanions and polycations in polyelectrolyte multilayer films, Macromolecules 37 (2004) 1159-1162.

[40] P. Nazaran, V. Bosio, W. Jaeger, D.F. Anghel, R.v. Klitzing, Lateral mobility of polyelectrolyte chains in multilayers, J. Phys. Chem. B 111 (2007) 8572-8581.
[41] R. Von Klitzing, Internal structure of polyelectrolyte multilayer assemblies, Phys. Chem. Chem. Phys. 8 (2006) 5012-5033.

[42] A.A. Antipov, G.B. Sukhorukov, E. Donath, H. Moehwald, Sustained release properties of polyelectrolyte multilayer capsules, J. Phys. Chem. B 105 (2001) 2281-2284.

[43] A. Fery, B. Schoeler, T. Cassagneau, F. Caruso, Nanoporous thin films formed by saltinduced structural changes in multilayers of poly(acrylic acid) and poly (allylamine), Langmuir 17 (2001) 3779-3783.

[44] J. Leddy, A.J. Bard, Polymer films on electrodes. Part XII. Chronoamperometric and rotating disk electrode determination of the mechanism of mass transport through poly(vinylferrocene) films, J. Electroanal. Chem. Interfacial Electrochem. 153 (1983) 223-242.

[45] T.H. Silva, S.V.P. Barreira, C. Moura, F. Silva, Electrochemical characterization of a self-assembled polyelectrolyte film, Port. Electrochim. Acta 21 (2003) 281-292.

[46] V.R Taliene, T. Ruzgas, V. Razumas, J. Kulys, Chronoamperometric and cyclic voltammetric study of carbon paste electrodes using ferricyanide and ferrocenemonocarboxylic acid, J. Electroanal. Chem. 372 (1994) 85-89.

[47] J.J. Harris, M.L. Bruening, Electrochemical and in situ ellipsometric investigation of the permeability and stability of layered polyelectrolyte films, Langmuir 16 (2000) 2006-2013.

[48] K. Haberska, O. Svensson, S. Shleev, L. Lindh, T. Arnebrant, T. Ruzgas, Activity of lactoperoxidase when adsorbed on protein layers, Talanta 76 (2008) 1159-1164.

[49] M. Gopinadhan, O. Ivanova, H. Ahrens, J.-U. Guenther, R. Steitz, C.A. Helm, The influence of secondary interactions during the formation of polyelectrolyte multilayers: layer thickness, bound water and layer interpenetration, J. Phys. Chem. B 111 (2007) 8426-8434.

[50] M. Landgren, B. Joensson, Determination of the optical properties of silicon/silica surfaces by means of ellipsometry, using different ambient media, J. Phys. Chem. 97 (1993) 1656-1660.

[51] J.A. De Feijter, J. Benjamins, F.A. Veer, Ellipsometry as a tool to study the adsorption behavior of synthetic and biopolymers at the air-water interface, Biopolymers 17 (1978) 1759-1772.

[52] F. Caruso, H. Lichtenfeld, E. Donath, H. Moehwald, Investigation of electrostatic interactions in polyelectrolyte multilayer films: binding of anionic fluorescent probes to layers assembled onto colloids, Macromolecules 32 (1999) 2317-2328.

[53] C.W. Hoogendam, J.C.W. Peters, R. Tuinier, A. De Keizer, M.A. Cohen Stuart, B.H. Bijsterbosch, Effective viscosity of polymer solutions: relation to the determination of the depletion thickness and thickness of the adsorbed layer of cellulose derivatives, J. Colloid Interface Sci. 207 (1998) 309-316.

[54] P. Sorlier, C. Rochas, I. Morfin, C. Viton, A. Domard, Light scattering studies of the solution properties of chitosans of varying degrees of acetylation, Biomacromolecules 4 (2003) 1034-1040.

[55] M. Kolasinska, R. Krastev, P. Warszynski, Characteristics of polyelectrolyte multilayers: Effect of PEI anchoring layer and posttreatment after deposition, J. Colloid Interface Sci. 305 (2007) 46-56.

[56] E. Poptoshev, B. Schoeler, F. Caruso, Influence of solvent quality on the growth of polyelectrolyte multilayers, Langmuir 20 (2004) 829-834

[57] J. Dejeu, L. Buisson, M.C. Guth, C. Roidor, F. Membrey, D. Charraut, A. Foissy, Early steps of the film growth mechanism in self-assembled multilayers of PAH and PSS on silica, Colloids Surf., A 288 (2006) 26-35.

[58] P.M. Biesheuvel, M.A.C. Stuart, Electrostatic free energy of weakly charged macromolecules in solution and intermacromolecular complexes consisting of oppositely charged polymers, Langmuir 20 (2004) 2785-2791.

[59] P.M. Claesson, B.W. Ninham, pH-dependent interactions between adsorbed chitosan layers, Langmuir 8 (1992) 1406-1412.

[60] R.v. Klitzing, J.E. Wong, W. Jaeger, R. Steitz, Short range interactions in polyelectrolyte multilayers, Curr. Opin. Colloid Interface Sci. 9 (2004) 158-162.

[61] J. Voeroes, The density and refractive index of adsorbing protein layers, Biophys. J. 87 (2004) 553-561.

[62] L. Lindh, I.E. Svendsen, O. Svensson, M. Cardenas, T. Arnebrant, The salivary mucin MUC5B and lactoperoxidase can be used for layer-by-layer film formation, J. Colloid Interface Sci. 310 (2007) 74-82.

[63] O. Svensson, K. Thuresson, T. Arnebrant, Interactions between chitosan-modified particles and mucin-coated surfaces, J. Colloid Interface Sci. 325 (2008) 346-350.

[64] D. Shoup, A. Szabo, Chronoamperometry at an ensemble of microdisk electrodes, J. Electroanal. Chem. Interfacial Electrochem. 160 (1984) 19-26.

[65] S.L. Petersen, D.E. Tallman, Gold and platinum poly(chlorotrifluoroethylene) composite electrodes, Anal. Chem. 62 (1990) 459-465.

[66] N. Mohtat, M. Byloos, M. Soucy, S. Morin, M. Morin, Electrochemical evidence of the adsorption of alkanethiols on two sites on $\mathrm{Ag}(111)$, J. Electroanal. Chem. 484 (2000) 120-130. 